\title{
NOTA
}

\section{DOSE LETAL DE RADIAÇÃO GAMA PARA OVOS DE Corcyra cephalonica (STAINTON, 1865) (Lepidoptera: Pyralidae), TRAÇA DO ARROZ}

\author{
J.A. DUARTE AGUILAR ${ }^{1}$; V. ARTHUR ${ }^{1}$ \\ Centro de Energla Nuclear na Agricultura/USP - C.P. 96 - CEP: 13400-970 - Piracicaba,SP
}

RESUMO: O. experimento teve como objetivo observar os efeitos da radiação gama em ovos da traça do arroz, Corcyra cephalonica (STAINTON, 1865). As doses utilizadas no experimento foram; $0 ; 25 ; 50 ; 75 ; 100 ; 125 ; 150$; $175 ; 200 \mathrm{~Gy}$. $O$ experimento foi conduzido em sala climatizada a $25 \pm 2^{\circ} \mathrm{C}$ e $70 \pm 10 \%$ de umidade relativa. Observou-se que as doses letais DL50 e DL100 para ovos provenientes de adultos criados em dieta artificial foram respectivamente de 16 e $75 \mathrm{~Gy}$.

Deacritores: radiação gama, traça do arroz, Corcyra cephalonica, dose letal

\section{LETHAL DOSE OF GAMMA RADIATION FOR EGGS OF Corcyre cephalonica (STAINTON, 1865) (LEPIDOPTERA:PYRALIDAE), RICE MOTH}

ARSTRACT: The aim of this experiment was to observe the effects of gamma radiation on rice moth Corcyra cephalonica (STAINTON, 1865) eggs. The doses utilized in this experiment were $0 ; 25 ; 50 ; 75 ; 100 ; 125 ; 150 ; 175$; $200 \mathrm{~Gy}$. The experiment was carried out in a climatic room at $25 \pm 2^{\circ} \mathrm{C}$ and $70 \pm 10 \% \mathrm{R}$.H. It was observed that lethal dose LD50 and LD100 for eggs from adults reared by artificial diet were 16 and $75 \mathrm{~Gy}$, respectively.

Key Words: gamma radiation, rice moth, Corcyra cephalonica, Iethal dose

\section{INTRODUÇÃO}

É possível o uso das radiações ionizantes na desinfestação de cereais e seus derivados como farinhas e farelos, nos quais se encontram ovos de traças, principalmente quando estes produtos são empacotados. Ovos e larvas jovens podem escapar de várias "screening" na operação de separação. Em pacotes pode ser feita a fumigação com produtos químicos que controlam principalmente adultos e larvas, ficando as vezes ovos para dar início a próxima geração do inseto praga. Além deste, outros métodos de controle de insetos depois de empacotado o produto não tem sido avaliados.

A Corcyracephalonica (Stainton, 1865) causa sérios danos durante a armazenagem, vindo a ser uma das mais importantes traças do arroz, nas regiões de clima tropical e subtropical, além de infestar cereais, farelos e farinhas ataca também amendoim, milho, arroz, cacau, café em grãos, soja, segundo KRISHNA \& AYYAR, (1934). Uma solução lógica segundo TILTON et al. (1974) para controlar ovos de traças em produtos empacotados, acondicionado em containers, é a irradiação com raios gama, pois é um método efetivo e econômico, não deixa resíduos $\mathrm{e}$ os insetos não adquirem resistência. Devido a isso, o objetivo do trabalho foi estudar a influência da radiação gama do Cobalto-60 em ovos de $C$. cephalonica.

\section{REVISÃO DE LITERATURA}

Segundo KAMEL \& HASSENEIN (1967), KAMEL et al. (1977), o periodo de preoviposição das fêmeas de $C$. cephalonica é de 24 horas, mostrando um período de oviposição de 2 a

\footnotetext{
${ }^{1}$ Bolsista do CNPq.
} 
7 dias e o número de ovos por fêmea é de 268 no inverno e 399 ovos no verão, dependendo da dieta em que são criadas. $O$ período de incubação dos ovos $\epsilon$ de 4 dias à $28,5^{\circ} \mathrm{C}$. HODGES (1979) cita que os ovos de Corcyra cephalonica, tem formato oval, com pequenas protuberâncias que medem aproximadamente $0,5 \times 0,3 \mathrm{~mm}$.

BROWER (1974) irradiou ovos de Plodia interpuctella (Hueb., 1813) com idade de 1-72 horas e mostrou que a idade dos ovos irradiados tem profunda influência na eclosão das lagartas. As doses de radiação gama usadas foram de $0,5 \mathrm{krad}$ a $50 \mathrm{krad}$, e o experimento mostrou que ovos de 18; 24; e 30 horas são igualmente sensíveis, e os de 24 horas foram selecionados como os mais radiosensíveis para induzir a esterilidade em adultos.

BECNZER\& FARKAS (1974) determinaram que os ovos são mais radiosensíveis, e que a dose de $35 \mathrm{krad}$ é suficiente para a desinfestação de produtos que contenham ovos e larvas de P.interpuctella.

BROWER (1975) irradiou ovos de $P$. interpuctella com diferentes idades, procurando determinar a indução de danos genéticos e a possibilidade de controle desta praga. Para ovos irradiados com dose de $2,0 \mathrm{krad}$ a eclosão de lagarta foi de $22,6 \%$.

CHAND \& SEHGAL (1978) irradiaram ovos de $C$. cephalonica de idades diferentes, 0-24, 24-48, 48-72 e 72-96 horas: para evitar a eclosão das lagartas de 72-96 e 24 horas a dose necessária foi de 5,0 e 3,5 krad, respectivamente.

RODRIGUES et al. (1981) determinaram as doses letais e esterilizante para ovos de Sitotroga cerealella (Oliv., 1819) e observaram ser de $10 \mathrm{krad}$ a dose letal para ovos.

ALLOTEY (1985) irradiou ovos de $C$. cephalonica de 1,2 e 3 dias e determinou que 25 krad foi a dose ideal para a desinfestação dos produtos.

ARTHUR (1985) observou que as doses letais para ovos de $S$. cerealella criadas em arroz e milho foram de 100 e $115 \mathrm{~Gy}$, respectivamente.

Ja TAMBORLIN (1988) observou que a dose letal para eliminar a totalidade dos ovos de $P$. interpuctella, foi de $125 \mathrm{~Gy}$.

\section{MATERIAL E MÉTODOS}

O presente trabalho foi desenvolvido no Laboratório da Seção de Radioentomologia do
Centro de Energia Nuclear na Agricultura, CENA SP - Piracicaba, São Paulo, em agosto de 1990, com ovos de C.cephalonica (Lepidoptera: Pyralidae), em sala climatizada com temperatura de $25-2^{\circ} \mathrm{C}$ e umidade relativa de $70-10 \%$, conforme registros diários em termohigrográfo.

O irradiador foi uma fonte de Cobalto60, tipo Gammabeam 650, com uma atividade de $2,91 \times 10 \mathrm{~Bq}$ e sob uma taxa de dose de 2,79 kGy/ hora para todos os tratamentos.

Para a coleta dos ovos, os adultos foram colocados em um vidro transparente, com capacidade de 3 litros, tampado com filó virado com a boca para baixo, para facilitar a coleta dos ovos e sua limpeza. Cada tratamento teve cinco repetições de 20 ovos cada, somando 100 ovos por tratamento com idade entre zero a 24 horas. Os ovos foram levados para serem irradiados, numa placa de Petri com tampa, medindo $2,5 \mathrm{~cm}$ de altura e $11,0 \mathrm{~cm}$ de diâmetro, em cujo interior foi colocado papel filtro quadriculado para facilitar a contagem da eclosão das lagartas.

As doses de radiação gama empregadas foram: 0 (testemunha), 25; 50; 75, 100; 125; 150; 175; 200 Gy. Para determinar a dose letal de radiação foi feita contagem sob lupa binocular, observando-se a viabilidade dos ovos. após a irradiação.

Esta contagem foi feita no 4.; 5.; 6 dias

\section{RESULTADOS E DISCUSSÃO}

Na TABELA 1 constam os valores numéricos, percentagem e média de ovos férteis e inférteis irradiados com doses crescentes de radiação gama, proveniente de adultos de Corcyra cephalonica, criadas em dieta articial.

Com esses dados construiu-se a Figura 1 , onde observamos as médias e variações numéricas de ovos férteis e inférteis irradiados com doses crescentes de radiação gama.

Considerando-se os efeitos da radiação gama observou-se que a dose suficiente para induzir a mortalidade total (DL100) dos ovos foi de 75 Gy (TABELA 1), enquanto que a dose para reduzir a eclosão das lagartas em 50\% ou seja (DL50) foi de $16 \mathrm{~Gy}$, determinada graficamente na Figura 1.

Os resultados obtidos neste experimento apresentam diferença dos determinados por CHAND \& SEHGAL (1978); provavelmente devido as condições nas quais o experimento foi realizado, podendo assim confirmar os resultados de BROWER (1974), concluindo que a idade dos ovos 
TABELA 1 - Viabilidade de ovos de Corcyra cephalonica (STAINTON, 1865), (Lepidoptera) irradiados com doses crescentes de radiação gama, criados em dieta artificial (média de 5 repetições).

\begin{tabular}{|c|c|c|c|c|c|c|}
\hline Dose (Gy) & Repetições & $\begin{array}{c}\mathbf{N}^{\circ} \text { ovos } \\
\text { irradiados }\end{array}$ & $\begin{array}{c}N^{\circ} \text { ovos } \\
\text { férteis }\end{array}$ & $\begin{array}{c}\text { \% ovos } \\
\text { ferteis }\end{array}$ & $\begin{array}{l}N^{\circ} \text { ovos } \\
\text { inférteis }\end{array}$ & $\begin{array}{l}\% \text { ovos } \\
\text { inférteis }\end{array}$ \\
\hline 0 & 5 & 20 & 17 & 85,0 & 3 & 15,0 \\
\hline 25 & 5 & 20 & 7 & 35,0 & 13 & 65,0 \\
\hline 50 & 5 & 20 & 1 & 5,0 & 19 & 95,0 \\
\hline 75 & 5 & 20 & 0 & 0,0 & 20 & 100,0 \\
\hline 100 & 5 & 20 & 0 & 0,0 & 20 & 100,0 \\
\hline 125 & 5 & 20 & 0 & 0,0 & 20 & 100,0 \\
\hline 150 & 5 & 20 & 0 & 0,0 & 20 & 100,0 \\
\hline 175 & 5 & 20 & 0 & 0,0 & 20 & 100,0 \\
\hline 200 & 5 & 20 & 0 & 0,0 & 20 & 100,0 \\
\hline
\end{tabular}

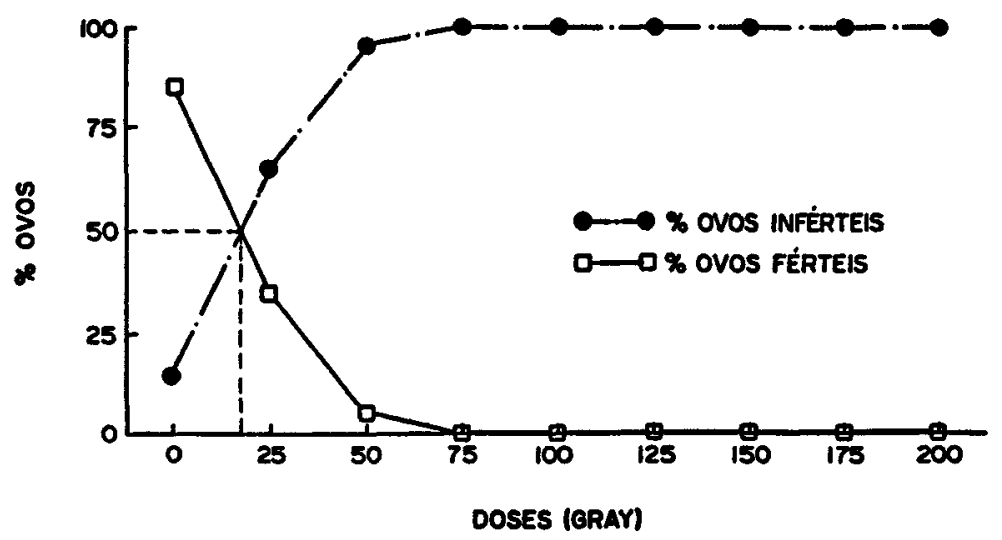

Figura 1. Porcentagem de ovos férteis e inférteis de Corcyra cephalonica (STAINTON, 1865) (Lepidoptera) irradiados com doses crescentes de radiação gama.

irradiados tem profunda influência na eclosão das lagartas. As doses utilizadas neste experimento são suficientes, tambem para causar a letalidade das lagartas e a esterilização de adultos provenientes de lagartas irradiadas, conforme os resultados obtidos por DUARTE-AGUILAR \& ARTHUR (1991).

\section{CONCLUSÕES}

As doses letais DL50 e DL100 de radiação gama para ovos de C.cephalonica, foram de $16 \mathrm{e}$ $75 \mathrm{~Gy}$, respectivamente.

\section{REFERENCIAS BIBLIOGRAFICAS}

ALLOTEY, J. Study of rediosensitivity of the immature stages of Corcyra cephalonica. Insect Science and its Application, Elmsford, 6(5):621-625, 1985. Apud Review of Applied Entomology. Serie A: Agricultural, Slough, 74(3):129, 1986. (Resumo).

ARTHUR, V. Efeitos esterilizantes e letais das radiações gama nas diferentes fases do ciclo evolutivo de Sitotroga cerealella (Olivier, 1819) (Lepidoptera Gelechiidae) em arroz e milho. Piracicaba, 1985. 77p. Tese (Doutorado) - Escola Superior de Agricultura "Luiz de Queiroz"/Universidade de Săo Paulo. 
BECNZER, J.; FARKAS, J. Investigation into the radioresistance of Plodia interpuctella (HUB). Acta Phitopatology Academia Scientiarm, Budapest, 9(1/2):153-60, 1974. Apud Entomology Abstracts, Philadelphia, 6(11):204, nov. 1975. (Resumo).

BROWER, J.H. Age as a factor in determining radiosensivity of eggs Plodia interouctella. Environmental Entomology, College Park, v.3, n.6, p.945-946, 1974.

BROWER, J.H. Sterility of adult indians meal moth and their progeny reared from gamma irradiated eggs. Envirommental Entomology, College Park, v.4, n.5, p.701-704, 1975.

CHAND, A.; SEHGAL, S.S. Influence of egg-age on hatchability response of Corcyra cephalonica (Stainton) to various dosages of gamma irradiation. Journal of Experimental Biology, New Delhi, v.16, n.7, p.815-816, 1978.

DUARTE AGUILAR, J.A.; ARTHUR, V. Controle de lagarta de Concyra cephalonica (Stain.1865) (Lep., pyralidae) traça arroz, através da radiaçăo gama do Cobalto-60. Lavoura Arrozeira, Porto Alegre, 1991. (No prelo)

HODGES, R.J. A review of the biology and control of the rice moth, Corcyra cephalonica (Staint.) (Lep. galleridae). London: Tropical Products Institule, 1979, 20p. (Report of the Tropical Products Institute, 125).

KAMEL, A.H.E.; ALI, M.A.; EL-BISHLAWAY, H.M. Effect of flour constituents on certain biological aspects of rice moth, Corcyra cephalonica (Stainton) (Lep. Galleridae). Bulletin de la Societe Entomologique d'Egipte, Cairo, v.61, p.137-142, 1977.
KAMEL, A.H.E.; HASSENEIN, M.H. Biological studies on Corcyra cephalonica (Stainton) (Lep. galleridae). Bulletin de Ia Societe Entomologique d'Egipte. Cairo. v.51, p.175-196, 1967.

KRISHNA, P.N.; AYYAR, B.A. A very destructive pest of stored product in South India, Corcyra cephalonica Stainton (Lepidoptera). Bulletin of Entomological Research, London, v.25, p.155-169, 1934.

RODRIGUES, Z.A.; REGO, A.M.; OLIVEIRA, M.L.; FERREIRA, D. Effects of gamma radiation Cobalt 60 on eggs and adults of Sitotroga cerealella (Oliv., 1819) (Lep. Gelechiidae) in laboratory. Radiation Techniques and their Applications to Insect Pests, Vienna, v.29, p.5, 1981.

TAMBORLIN, M.J. Efeitos das radiações gama nas fases do ciclo evolutivo de Plodia interpuctella (Hueb, 1813) (Lep. pyralidae) em dieta artificial. Piracicaba, 1988. 91p. Dissertaçāo (Mestrado) - Escola Superior de Agricultura "Luiz de Queiroz"/Universidade de São Paulo.

TILTON, E.W.; JOHN, H.; BROWER, J.H.; COGBURN, R.R. Gamma irradiation for control of insects in wheat flour. Journal of Economic Entomology, College Park, v.67, n.1, p.430-432, 1974.

Enviado para publicação em 04.08 .93

Aceito para publicação em 03/09/93 